\title{
The Existing Study and Future Directions on the Connotation and Measurement of Sense of Responsibility
}

\author{
Shenghua Zhang* \\ General Platform Department, Beijing Information Technology College \\ No. 5 Fangyuan West Road, Chaoyang District, Beijing, People's Republic of China 100016 \\ zhangsh@bitc.edu.cn \\ ${ }^{*}$ Corresponding author
}

Keywords: Quality Education, Sense of Responsibility, Measurement.

\begin{abstract}
How to cultivate the sense of responsibility of the students is an important issue for educators. This paper reviewed the literatures and researches on sense of responsibility and analyzed the current study domestic and abroad from three aspects: the connotation of sense of responsibility, the structure of sense of responsibility, and the measurement of sense of responsibility. The study analyzed the advantages and disadvantages of the relevant theories concerned with the connotation and the structure of sense of responsibility and sorted out the methods of sense of responsibility measurement. This study provides a clear theoretical method and guide for the study on sense of responsibility.
\end{abstract}

\section{Introduction}

In recent years, people have talked a lot about the lack of sense of responsibility of young people. For the school education, educators should try to find out the reasons and the solutions to the issue.

In order to solve this problem, we need to make clear of what sense of responsibility mean for education. We discuss this problem in two aspects. First, in the new era, with the economic and social progress and development, what is the sense of responsibility of the talents required by the society? This is to define the goals and content of education. Second, what is the criteria of sense of responsibility or how to measure sense of responsibility? This is about quality evaluation of education. These two aspects are the key and essential issues in talent training and education. It is, definitely, the super concern of this study. The study reviewed the corresponding literature and sorted out the corresponding research made by scholars domestic and abroad.

\section{Defining Sense of Responsibility}

According to the existing knowledge of sense of responsibility, the widely accepted opinions are: sense of responsibility is not only a common concept talked a lot in people's daily lives, it is also the focus of research in the fields of sociology, education and psychology; sense of responsibility is not only an important factor in maintaining social harmony and promoting social civilization, it is also a positive personality trait for individual development as well; sense of responsibility is the concentrated reflection of the individual's various relationships of responsibilities in real life and it is a kind of psychological cognition which is the internalized and stable cognition of the requirements of the society.

The researches on sense of responsibility made by western scholars are mainly focused on social behavior, moral judgment, responsibility attribution, etc. In the 1930s, psychologist Piaget introduced responsibility to the field of moral judgment and considered it as the core content of the children's moral judgment development process [1]. French sociologist Paul Fauconnet wrote in his doctoral thesis that sense of responsibility is a psychological trait which belongs to the active recipient who chooses punishment according to the norms and rules [2]. In the late $20^{\text {th }}$ century, Western scholars made great progress in this filed. Schlenker held the idea that sense of responsibility refers to the process in which individuals fulfill their responsibility for society and 
others by making commitment and performing duties that conform to social norms [3]. Lerner believed that responsibility is a dominant or implicit psychological expectation related to his or her own cognition, emotion, and behaviors which is generated when individuals evaluate others' behavior [4]. Andrew et al. believe that responsibility is a state in which individuals are responsible for their own decisions and behaviors [5]. Later researchers believed that sense of responsibility is the individual's tendency to fulfill or comply with social norms and is a psychological and behavioral process affected by situations or events and triggered by the internalized behavioral norm or self-regulation [6]. In a word, for the western scholars, psychological research on sense of responsibility are focused more on the attribution of sense of responsibility and less on the moral responsibility obligations.

The domestic scholars hold that sense of responsibility is an important quality of a person's psychological personality and it is a person's positive attitude toward the behavior norms of the society and toward the tasks he undertakes. Zhang Jijia (1998) defined sense of responsibility as individual's self-consciousness of one's responsibility and the behavioral tendency of actively performing the duties [7]. Li Xue (2004) held that sense of responsibility is a kind of individual psychological quality that reflects the individual's commitment to social obligations in social activities [8]. Zhao Xingkui and Zhang Dajun (2007) held that sense of responsibility is a static quality expressed through the dynamic form of responsibility cognition, responsibility personality and responsibility adaptation [9]. Li Ming and Ye Haosheng(2009) held that sense of responsibility includes three inseparable aspects, that is: responsibility quality, responsibility emotion and responsibility cognition, and it is the unity of psychological tendency, psychological activity and psychological content that affects responsible behavior [10].

Domestic scholars focused their research mainly on psychological mechanisms of sense of responsibility, the framework of sense of responsibility, and the essential characteristics of sense of responsibility. They hold that sense of responsibility is an important part of the psychological trait of one's personality and is the psychological phenomenon embodied in individual psychological activities and the important content of personal psychological activities. It is the psychological tendency of the individual who responds to the requirements of the society by active cognitive processing.

In summary, for the study of sense of responsibility, although western scholars have different understandings and applications in different fields, they have something in common, that is: they care much about responsibility cognition and behavioral consequences; they hold that sense of responsibility is related to people's conscious activities; they pay little attention to such factors as beliefs and values. In studying sense of responsibility, domestic scholars pay attention not only to the formation mechanism of individual psychological quality, but also to the impact of social requirements on the individual's psychological quality. Thus, the definition of sense of responsibility given by domestic scholars reflects the dual attributes of individual and social development. And it is more general than that given by the scholars abroad.

\section{The Structure of Sense of Responsibility}

The study of the structure of sense of responsibility is an important area of psychological research. Influenced by different theoretical schools, there are different methods of how to construct the structure of sense of responsibility. The current theoretical research abroad on sense of responsibility mainly includes the following kinds: moral responsibility theory represented by Piaget, Kohlberg, and Lind; personality responsibility theory represented by Digman, Costa and McCrae; cognition responsibility represented by Schlenker. In addition, domestic scholars have also done some in-depth study of the structure of sense of responsibility and arrived at some positive understanding, such as the integrated responsibility theory (three-sided model) proposed by Li Ming and Ye Haosheng.

Domestic and foreign scholars studied two types of the structure of responsibility from the value orientation perspective. One is based on personality traits. The other is based on situational responsibility. 
First, for the theories based on personality traits.

Zhu Zhixian (1989) divided sense of responsibility into three dimensions: responsibility awareness, responsibility emotion and responsibility behavior [11]. Zhang Jijia (1998) held that sense of responsibility consists of five dimensions: responsibility awareness, responsibility emotion, responsibility behavior, responsibility motivation and the ability for fulfilling responsibilities [12]. Wang Yan (2003) divided sense of responsibility of college students into six dimensions: self-responsibility, sense of family responsibility, responsibility for others, professional responsibility, collective responsibility and social responsibility [13].

The dimensions of sense of responsibility defined by Costa (1992) are self-confidence, organized, dependability, pursuit of achievement, self-Regulation, thoughtful, capability [14]. Rachman (1995) divided sense of responsibility into four dimensions, that is, responsibility for injury, responsibility in a social context, positive perception of responsibility, and a combination of cognition and action [15]. In the AB5C personality model (abridged Big Five dimensional circumflex), theoretically, there are nine dimensions of responsibility traits: due diligence, responsiveness, efficiency, prudence, loyalty, rigorous rationality, firmness, decisiveness, perfection, orderliness, and compliance with norms and rules [16].

In summary, researchers at home and abroad arrived at consensus that from the psychological process perspective, sense of responsibility should at least include three aspects: responsibility cognition, responsibility emotion and responsibility behavior. The three aspects interact with each other to form the overall sense of responsibility. Sense of responsibility is an important psychological quality with a complex structure. Studying sense of responsibility from a single dimension cannot reflect the full picture of sense of responsibility. We can approach sense of responsibilities from multiple dimensions.

The following are typical theories based on situational responsibility.

Schlenker (1999), who proposed the triangle model of accountability, held that sense of responsibility consisted of three key elements: specific event, behavior norms and the identity of individuals [17]. Under certain situations, the three elements interact with each other, which reflects the individual's value judgment about responsibility. Auhagen (2001) confined the study of sense of responsibility to daily life. It is a specific situation in life in which people feel responsible, which involves cognition, emotion, motivation, and goals, etc. [18]. Li Ming and Ye Haosheng proposed the three-sided structure theory of sense of responsibility. According to the three-sided theory, sense of responsibility consists of responsibility quality, responsibility emotion and responsibility cognition and the three sides interact with each other and influence the responsible behavior [19]. Kuang Zhihua (2012) held that the psychological structure of sense of responsibility is a one-dimensional decision model consisting of responsibility cognition, responsibility emotion and responsibility behavior [20].

In addition to research on the structure of sense of responsibility from the perspective of personality traits and relevant situations, the other researchers distinguish sense of responsibility from the perspective of levels and dimensions. Due to space limitations, we will not repeat them here.

According to the research made by domestic and foreign scholars, we can draw the following conclusions.

First of all, due to the difference in theoretical construction and research methods, researchers at home and abroad still have some divergence in the study of the psychological structure of sense of responsibility; there is also great disagreement on the understanding of responsibility, responsibility emotion and responsibility behavior. In addition, the difference of the subjects involved in the research, and the difference of the research perspective and the degree of abstraction are also important factors for the disagreement.

Secondly, the study of the structure of responsibility is mostly static, which means the mechanism of the influence of psychology on responsibility behavior cannot be well explained. In the current study of sense of responsibility structure, time factor is not included in the relevant theoretical models. Therefore, future research should be made to approach the structure of sense of 
responsibility from a dynamic and developing perspective and to explore the structure of sense of responsibility innovatively.

Finally, considering the complexity of the structure of responsibility, the demographic variables of the subjects will also affect the results of the research. These elements are also worth further study.

\section{The Measurement of Sense of Responsibility}

Sense of responsibility is one of the most important differentiating variables in psychology research and application. The measurement of sense of responsibility is important for assessing individual personality traits. It is also the general concern in the field of psychology research. The measurement of sense of responsibility can be traced back to the single-dimensional moral judgment experiment conducted by Piaget and Kohlberg respectively. They used the dual story method and the moral dilemma to measure children's stages of moral development.

Based on the behavioral performance, follow-up researchers at home and abroad developed some sense of responsibility scales. The scales commonly used in the application research of sense of responsibilities are: a Personality Scale for Social Responsibility 1952 by Gough [21]; a Scale for Measuring Attitudes of Social Responsibility in Children 1957 by Harris [22]; Personal Responsibility Scale 1987 by Martel [23]; The Job Responsibilities Scale 1999 by Ludlow \& Lunz [24]; Student Personal Responsibility Scale-10 2001 by Sangeeta [25].

Based on behavioral tests, domestic scholars developed several responsibility measurement scales, for example, the Questionnaire of Middle School Students' Social Responsibility 2004 by Li Xue. Using qualitative research methods, Jiang Yong and Pang Lijuan compiled the Children's Responsibility Questionnaire 2000 and then the teacher gave the evaluation according to their daily observation on the children [26].

Although the above-mentioned measurement scale on responsibility developed by domestic and foreign scholars has been widely used, and researchers has obtained a large number of measurement data of individual subjects, on particular occasions, these methods may have a social desirability effect and there may also be false problems, and thus the reliability and validity of the scales will be affected. Therefore, researchers developed the implicit test method of sense of responsibility. Based on Thematic Apperception Test (TAT), Winter developed the psychodynamic method for sense of responsibility measurement [27]. Using the standard Implicit Association Test (IAT) program, Steffens created a sense of responsibility IAT test [28]. Greenwald proposed implicit measurement of the responses for responsibilities, that is, Multifactor Traits Implicit Association Test (MFT-IAT) [29].

Although the implicit test method avoids certain defects of the scale measurement, whether it is scientific and rational or not still needs further verification. For example, subjects were given the corresponding fuzzy stimuli and then the scholar analyzes their free responses. Can the results be used to infer the true subconscious mind of the subjects? With the continuous development of psychological research techniques, some scholars have tried to use the method of neuroimaging to measure sense of responsibility. Vincent uses neuroimaging to study the neural basis of moral responsibility and then to determine a person's common core mental capacity of being responsible or moral. Then researchers use this knowledge to assess a particular individual's sense of responsibility [30] [31].

By reviewing the previous research, we find that in the field of responsibility measurement, there are still many issues need further research. First, the behavioral test methods for the measurement of sense of responsibility lack sufficient ecological validity. Second, research based on daily experience for sense of responsibility, are focused on analyzing the particular situation in which people feel responsible. Although researchers explored and verified the psychological structure model of sense of responsibility and the individual's cognition characteristics for responsibility, the empirical research was not enough. Third, to some extent, the implicit test method of sense of responsibility has a high degree of credibility. However, it is of great difficulty of operating and there is not enough empirical research to prove its high validity [32]. Fourth, although the 
neuroimaging method are reliable and valid, there are still two shortcomings in neuroimaging measurement method. One is the ignorance of the influence of social factors and the direct influence of brain stimuli. The other is that the current researches about sense of responsibility focuses mainly on general groups and researches for specific groups is not sufficient.

\section{References}

[1] Jean Piaget, The moral judgement of the child, Shandong Education Press, 1984, First Edition.

[2] Paul Fauconnet, La responsabilité: Etude de sociologie, Paris: Alcan, 1920.

[3] [17] B. R. Schlenker, T. W. Britt, J. Pennington, R. Murphy, and K. Doherty, The triangle model of responsibility, Psychological Review, vol.101(4), pp. 632-652, 1994.

[4] J. S. Lerner, and P. E. Tetlock, Accounting for the effects of accountability, Psychological Bulletin, vol.125(2), pp. 255-275, 1999.

[5] Q. Andrew, and B. R. Schlenker, "Can accountability produce independence? Goals as determinants of the impact of accountability on conformity, Personality and Social Psychology Bulltin, vol.28(26), pp. 472-483, 2002.

[6] [18] A. Auhagen, and H. Bierhoff, Responsibility at the beginning of the third millennium. In A. Auhagen \& H. Bierhoff (Eds.), Responsibility: the many faces of a social phenomenon. Routledge, pp. 61-67, 179-184, 2001.

[7] [12] Jijia Zhang, On the psychological structure of sense of responsibility, Educational Research and Experiment, pp. 43-47, 1998(4).

[8] Xue Li, Study on the social responsibility structure and its development characteristics of middle school students, Southwest Normal University, Chongqing, 2004.

[9] [24] Xingkui Zhao, and Dajun Zhang, Meaning and structure of responsibility and sense of responsibility, Journal of ShanXi Finance and Economics University (higher education edition), Vol .10 (1), pp. 22-25, 2007.

[10][19] Ming Li, and Haosheng Ye, The multiple connotation and structure of responsibility and the relevant theoretical integration, Psychological Development and Education, pp. 123-128, 2009(3).

[11]Zhixian Zhu, Psychology encyclopedia, Beijing Normal University Press, pp. 930, 1989.

[13]Yan Wang, A Report on the sense of responsibility of modern university students, Youth Study, pp. 18-23, 2003(1).

[14]R. R. Mccrae, and P. T. Costa, A five-factor theory of personality, Journal of Investing, pp. 139-153, 1999.

[15]S. Rachman, D. S. Thordarson, R. Shafran, and S. R. Woody, Perceived responsibility: structure and significance, Behavior Research and Therapy, vol. 33(7), pp. 779-784, 1995.

[16]W. K. Hofstee, R. B. De, and L. R. Goldberg, Integration of the big five and circumflex approaches to trait structure, J Pers Soc Psychol, vol. 63(1), pp. 146 -163, 1992.

[20]Zhihua Kuang, The mental framework of responsibility: An Everyday-Experience Approach Study, Journal of Psychological Science, vol. 35(2), pp. 430-435, 2012.

[21]H. G. Gough, H. Mcclosky, and P. E. Meehl, A personality scale for social responsibility, Journal of Abnormal \& Social Psychology, vol.47 (1), pp.73 -80, 1952.

[22]D. B. Harris, A scale for measuring attitudes of social responsibility in children, Journal of Abnormal \& Social Psychology, vol.55 (3), pp .322 -326, 1957. 
[23] J. Martel, S. J. Mckelvie, and L. Standing, Validity of an intuitive personality scale: personal responsibility as a predictor of academic achievement, Educational and Psychology Measurement, vol. 47(4), pp. 1153-1163, 1987.

[24]L. H. Ludlow, The structure of the job responsibilities scale: a multimethod analysis, Educational and Psychological Measurement, vol. 59(6), pp. 962-975, 1999.

[25]S. Singg, and J. A. Ader, Development of the student personal responsibility scale-10, Social Behavior and Personality, vol. 29(4), pp. 331-335, 2001.

[26] Yong Jiang, and Lijuan Pang, The EFA and CFA on the Structure of Dimensions of Children's Responsibility, Psychological Science, vol. 23(4), PP. 417-420, 2000.

[27]D. G. Winter, and N. B. Barenbaum, Responsibility and the power motive in women and men, Journal of Personality, vol. 53(2), pp. 335-355, 2010.

[28]M. C. Steffens, and S. S. König, Predicting spontaneous big five behavior with implicit association tests, European Journal of Psychological Assessment, vol. 22(1), pp. 13-20, 2006.

[29]A. G. Greenwald, Method for measuring strength of associations of multidimensional traits, pp. 45-74, 2007.

[30]N. A .Vincent, Responsibility, dysfunction and capacity, Neuroethics, vol. 1(3), pp. 199-204, 2008.

[31]N. A. Vincent, Neuroimaging and responsibility assessments, Neuroethics, vol. 4(1), pp. 35-49, 2011.

[32]Zuwei Yu, Biyun Wu and Yi Ren, The current study and future outlook on the study of responsibility, Journal of Inner Mongolia Normal University (Educational Science), vol. 28(8), pp.54-60, 2015. 